\title{
Self-Measurement of Blood Pressure at Home in the Management of Hypertension
}

\author{
Hilde Celis, MD; Elly Den Hond, PhD; and Jan A. Staessen, MD, PhD, FAHA
}

\begin{abstract}
To be suitable for the management of hypertension, self-measurement of blood pressure (BP) at home should follow international recommendations. The use of accurate and validated measuring devices is an important prerequisite. Upper arm BP monitors are the first choice, while wrist and finger devices cannot be recommended. Measurements should, preferentially, be downloaded from the memory of a device or printed. Reference values have been proposed, but were mostly based on cross-sectional observations and have not yet been widely validated by prospective outcome studies. Currently, levels of home BP of $<135 \mathrm{~mm} \mathrm{Hg}$ systolic and $85 \mathrm{~mm} \mathrm{Hg}$ diastolic are usually considered normal. Home BP measurement is sometimes recommended as an alternative to ambulatory BP monitoring to diagnose white-coat hypertension. However, home BP measurement cannot replace ambulatory BP monitoring in the diagnosis of hypertension (white-coat), but both techniques have complementary roles. The appropriateness of home BP measurement to guide antihypertensive treatment has only been tested in one large-scale randomized trial: the THOP (Treatment of Hypertension Based on Home or Office Blood Pressure) trial. The THOP trial showed that antihypertensive treatment based on home instead of office BP led to less intensive drug treatment, but also to less BP control with no differences in general wellbeing and left ventricular mass. Home BP monitoring also contributed to the identification of patients with white-coat hypertension. On balance, most evidence supports the view that office BP measurement remains the key in the diagnosis and treatment of hypertension. Treatment can be started without confirmation of elevated office BP in patients with high office BP and target organ damage, or a high cardiovascular risk profile. In patients with raised office BP but without target organ damage (white-coat hypertension), or with normal office BP but unexplained target organ damage (masked hypertension), ambulatory or home BP monitoring or both must be used to confirm the diagnosis. Few longitudinal studies have addressed the long-term prognostic meaning of home BP measurement. Until more prospective data become available, management of hypertension exclusively based on self-measurement of BP at home cannot be recommended.
\end{abstract}

Keywords: Blood pressure; Blood pressure determination; Blood pressure monitoring, ambulatory; Hypertension; Self care

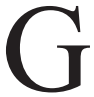 uidelines for Self-Measurement of Blood Pressure at Home}

Advantages of self-measurement of blood pressure

Self-measurement of blood pressure (BP) at home can accomplish several of the advantages of ambulatory BP monitoring, such as a greater number of readings, an avoidance of the white-coat syndrome and, when automated

Reprint Requests: Hilde Celis, MD, U.Z. Gasthuisberg - Dienst Hypertensie, Herestraat 49, 3000 Leuven - Belgium, Telephone: +32-|6-34363। Fax: +32-16-343766, Email: hilde.celis@med.kuleuven.ac.be devices are used, an absence of observer bias. Furthermore, self-measurement of BP may also increase compliance with antihypertensive therapy and reduce the number of visits required for the diagnosis and treatment of hypertension. It is also a less expensive method of monitoring blood pressure. 1,2

Requirements for accurate home BP measurement

Self-measurement of BP at home can only become a useful

Received: September 21, 2004

Revised: December 29, 2004

Accepted: January 17, 2004 
instrument in the management of hypertension if the application of the technique is well standardized and complies with defined quality criteria. Most national and international hypertension guidelines include some recommendations for home BP measurement. ${ }^{3-6}$ In principle, these guidelines are not different from those of BP measurement in general, but some points deserve special attention.

- Only accurate and properly validated BP measuring devices should be used. Any device, irrespective of whether it is employed in a clinical setting or in research, should have passed independent validation according to at least one of three generally accepted protocols: 1) the protocol of the British Hypertension Society (BHS), 2) the protocol of the Association for the Advancement of Medical Instrumentation (AAMI), or 3) the international protocol..$^{3,7-11} \mathrm{~A}$ device can only be considered as validated after a positive report has been published in a peer reviewed journal.

- Different types of BP monitors for self-measurement are available. Using auscultatory or aneroid devices might provide reliable measurements, but have several major drawbacks: proper instruction of the patients can take up to 45 minutes, ${ }^{9}$ aneroid devices are susceptible of becoming inaccurate without this being apparent to the user ${ }^{8}$ and, due to the known toxic effects of mercury on the environment, the mercury sphygmomanometer might well be banned from clinical use in the near future. ${ }^{3,9}$ Consequently, fully automated, electronic (almost exclusively oscillometric) devices are presently the most commonly marketed. Experts recommend upper arm devices for home BP measurement, while the use of wrist and finger devices is discouraged. $3,5,9,10$ Wrist devices, although easier to use by the patient, ${ }^{10}$ have a number of shortcomings. 9,10 Most of the wrist devices have not passed independent validation, ${ }^{11-13}$ although some have at least undergone some validation. ${ }^{14,15}$ Wrist monitors are more error prone than upper arm devices because the measurements are more likely to be influenced by anatomical factors, such as wrist anatomy $^{11}$ and dorsal or ulnar flexion of the wrist, $3,11,15$ as well as by the hydrostatic pressure of the blood if the wrist is not correctly positioned at the heart level. 3,15 To overcome the latter problem, a wrist home BP device with a position sensor has recently been developed and tested; ${ }^{14}$ however, its performance in clinical practice needs further evaluation. Finger devices share some of the shortcomings of wrist devices, such as the effect of limb position on measured pressure. Furthermore, inaccuracies can occur because of measurement distortion with peripheral vasoconstriction and alteration of blood pressure due to the more distal site of the recording. ${ }^{3}$ A regularly updated list of independently tested and validated BP devices for self-measurement of BP at home ${ }^{8}$ is available at the web site of the British Hypertension Society (http://www.hyp.ac.uk/bhs/bp_monitors/automatic.htm) and at the non-profit educational web site $\mathrm{dabl}{ }^{\circledR}$ Educational Trust (http://www.dableducational.com).

- As for office and ambulatory BP measurement, cuffs of appropriate size should be used (small adult or child cuff with bladder $12 \mathrm{~cm}$ x $18 \mathrm{~cm}$ for arm circumference $<23 \mathrm{~cm}$, standard adult cuff with bladder $12 \mathrm{~cm} \mathrm{x} 26$ $\mathrm{cm}$ for arm circumference $<33 \mathrm{~cm}$, and large adult cuff with bladder $12 \mathrm{~cm} \mathrm{x} 40 \mathrm{~cm}$ for arm circumference up to $50 \mathrm{~cm}) .3,6$

- Patients should rest 5 minutes in the sitting position before starting measurement.

- Home BP monitoring should always be performed under medical supervision. Patients should not titrate or change their antihypertensive drug treatment unless advised to do so by their doctor.

- Patient diaries and reports may be unreliable. Experts therefore recommend the use of devices that can be interfaced with a printer or a computer to produce a record of the readings with documentation of date and time of the day.

\section{Self-Measurement of BP at Home: How Often?}

Experts have not yet reached a general consensus about a standard protocol (how many measurements and on how many days) patients should follow to measure their BP at home. In a recent editorial in the Journal of Hypertension, Parati et al. ${ }^{16}$ describe two different methods to define the most suitable schedule for home BP measurement, namely a statistical and a clinical approach. In the statistical approach, the reproducibility and stability over time of home BP values and their relation with ambulatory BP values are used as criteria for defining the best frequency of home BP measurements. The clinical approach is based on the power of home BP values to predict cardiovascular outcome.

\section{Statistical approach}

The reproducibility of home BP depends on the number of measurements. It has been reported to be best if the average home BP is derived from 30 measurements, ${ }^{17}$ but other investigators found that no further improvement is obtained when increasing the number above 5 or 6 home BP measurements. ${ }^{18,19}$ Furthermore, also excluding the first day of measurements seems to improve reproducibility. 20,21 Stergiou et al. ${ }^{20}$ also showed that to obtain reliable and reproducible values at least two monitoring days were needed. Our group tried to define the best measurement schedule by looking at the stability of home BP measurements over time and calculated that at least 3 days of home BP measurements are needed to obtain stable BP values. ${ }^{22,23}$ On the other hand, Brook ${ }^{24}$ reported that if the accuracy of the average home BP was determined by agreement with average ambulatory BP values, the total number of measurements and total duration of monitoring were not important and that most benefits of home BP 
monitoring could be achieved by obtaining as few as 2 home BP measurements on one day. The advantage of home BP monitoring thus does not seem only to lie in the statistical advantages associated with the availability of a lot of measurements, but also in obtaining information on BP levels out of the clinical setting with even a few home BP measurements. ${ }^{16}$

\section{Clinical approach}

More recently, Ohkubo et al. ${ }^{25}$ tried to identify the best frequency of home BP measurements based on prognostic data from the Ohasama study, a population study carried out in a rural community in Japan. The office BP was measured twice at the screening visit and the subjects were asked to measure their BP at home once a day for a maximum of 4 weeks. Ohkubo and colleagues showed the predictive value for the risk of stroke of the average home BP measurement increased progressively, and without any threshold, if the number of measurements was increased from 1 to 14 . This study also demonstrated that there was only a modest additional benefit by averaging all available measurements. The authors concluded that as many measurements as possible (preferably more than 14) are recommended for the best prediction of stroke risk. However, the results of this study should be interpreted with caution, since compared to most other studies, the Ohasama study had a rather unusual measurement schedule for home BP with only one single morning measurement.

\section{Recommendation}

For diagnostic purposes, some experts ${ }^{3,6}$ propose duplicate measurements every morning and evening during a 7-day period, sometimes with exclusion of the readings of the first day. Although evidence based on statistical and clinical approaches seems to suggest fewer measurements may offer similar benefits to taking several, the reproducibility of home BP based on a limited number of measurements, as suggested by the statistical approach, might be inadequate for decision making in the individual patient. Since home BP monitoring is relatively inexpensive and easily accepted by patients, it is reasonable to recommend taking more home BP readings than the statistically reliable minimum number.

\section{Definition of Normal Values}

Definition of normality based on cross-sectional data Several investigators proposed reference values based on cross-sectional observations but used different statistical criteria. ${ }^{26-29}$ Therefore, in an attempt to derive a generally accepted reference frame for the home BP, we pooled all available data published through the end of 1996 into a meta-analysis involving 17 studies and 5,422 subjects. ${ }^{30}$ Eight of these studies included normotensives as well as untreated hypertensives. The nine other studies included only normotensives. We applied four different methods to derive a reference frame for the self-measured BP at home. In all normotensive subjects (office BP $<140 \mathrm{~mm} \mathrm{Hg}$ systolic and $<90 \mathrm{~mm} \mathrm{Hg}$ diastolic), we computed the $95^{\text {th }}$ percentile and the mean +2 standard deviations (SD) of the home BP as the upper threshold of normality (distribution method). Two alternative approaches started from subjects not preselected on the basis of their office BP. In the percentile method, we calculated the percentile ranks of an office BP of $140 \mathrm{~mm} \mathrm{Hg}$ systolic or $90 \mathrm{~mm} \mathrm{Hg}$ diastolic, and we determined the values of the home BP corresponding with these ranks. Finally, the values of the home BP equivalent to an office BP of $140 \mathrm{~mm} \mathrm{Hg}$ systolic and $90 \mathrm{~mm} \mathrm{Hg}$ diastolic were deduced by means of regression analysis (regression method). The reference values determined by the distribution methods, i.e., the mean $+2 \mathrm{SD}$ and the $95^{\text {th }}$ percentile of the distribution, in normotensives were 137/89 $\mathrm{mm} \mathrm{Hg}$ and 135/86 $\mathrm{mm} \mathrm{Hg}$, respectively. The cutoff points derived by the percentile and regression methods were considerably lower: 129/84 mm Hg and $125 / 79 \mathrm{~mm} \mathrm{Hg}$.

We also attempted to determine reference values for the self-measured BP at home using the individual data available in the international database of self-recorded BP in normotensive and untreated hypertensive subjects. ${ }^{31}$ This database includes 4,668 untreated subjects recruited by 13 research groups. To define operational thresholds for the home BP, we only used the data for the 2,401 subjects who were normotensive on office BP measurement. The reference values for the home BP determined from the $95^{\text {th }}$ percentiles of the BP distributions in these normotensive subjects were $139 \mathrm{~mm} \mathrm{Hg}$ systolic and $85 \mathrm{~mm} \mathrm{Hg}$ diastolic.

Subsequently, Stergiou et al. ${ }^{32}$ conducted a cross-sectional population survey, the Didima study, to determine normal thresholds for self-measured BP. After the exclusion of the treated hypertensives, data from 526 subjects were analyzed. If the $95^{\text {th }}$ percentiles of the home BP distributions in 476 normotensive subjects were used, the upper limit of normality was a self-measured BP of $140 \mathrm{~mm} \mathrm{Hg}$ systolic and $83 \mathrm{~mm} \mathrm{Hg}$ diastolic. The cutoff points derived by the percentile and regression methods where 140/86 $\mathrm{mm} \mathrm{Hg}$ and 137/83 mm Hg.

\section{Definition of normality based on outcome data}

A normal BP should ideally be determined in terms of cardiovascular risk. In the previously mentioned Ohasama study, ${ }^{25,33}$ the self-measured BP at home (mean of 3 to 38 measurements) was a better predictor of total mortality than office BP measured at screening. The Ohasama investigators were also the first to propose reference values for self-measurement of BP at home based on prognostic criteria. ${ }^{34}$ They found a linear relationship between systolic home BP and mortality, and subsequently set the upper norm for systolic self-measured BP at home at $137 \mathrm{~mm} \mathrm{Hg}$. The association between diastolic home BP and mortality was U-shaped. The Ohasama group considered diastolic self-measured BP values at home between 66 and $83 \mathrm{~mm} \mathrm{Hg}$ as normotensive, $\geq 84 \mathrm{~mm} \mathrm{Hg}$ as hypertensive and values below $66 \mathrm{~mm} \mathrm{Hg}$ as abnormally low. 
More recently the final results of the SHEAF (Self-measurement of Blood Pressure at Home in the Elderly: Assessment and Follow-up) study were published. ${ }^{35}$ Bobrie and colleagues evaluated the prognostic value of the self-measured BP at home and the office measured BP in a 3 -year prospective cohort study in a French population of treated hypertensive patients. They showed that the home BP, but not the office BP, was predictive of cardiovascular events, but neither measurement method was significantly related to cardiovascular events or total mortality. The cardiovascular prognosis of patients with controlled hypertension (normal office and home BP) or isolated office hypertension (high office BP, normal home BP) was comparable. In contrast, those with masked hypertension (normal office BP, high home BP) had a worse prognosis similar to that of the uncontrolled hypertensives (high office and home BP). Although the SHEAF investigators had proposed 135/85 $\mathrm{mm} \mathrm{Hg}$ as an operational threshold for normotension based on a retrospective analysis of their baseline data in an earlier publication, ${ }^{36}$ their final publication ${ }^{37}$ did not contain a new proposal based on the prognostic data.

\section{Current definition of normality}

Most expert committees and national and international guidelines committees concur with the idea that levels of the self-measured BP at home lower than $135 \mathrm{~mm} \mathrm{Hg}$ systolic and $85 \mathrm{~mm} \mathrm{Hg}$ diastolic are normal. ${ }^{3-6,9,30,38,39}$ However, more prospective studies are urgently needed to establish with more certainty the normal range of the self-measured home BP, including the operational threshold at which drug treatment should be instituted.

\section{Self-Measured BP at Home and the Diagnosis of Hypertension}

Recent hypertension guidelines ${ }^{3,39}$ propose the use of self-measurement of BP at home as an important means to evaluate the response to antihypertensive treatment, to improve compliance with therapy and, most importantly, as an alternative for ambulatory BP measurement to confirm or refute the diagnosis of white-coat hypertension. Instead of using either ambulatory or self-measurement of BP, some investigators have proposed to use an algorithm combining both techniques. ${ }^{23,40,41}$ According to this algorithm, patients with persistently high office BP values and no evidence of target organ damage should engage in self-monitoring of their BP, and if this demonstrates normal values, ambulatory $\mathrm{BP}$ monitoring is used to confirm the diagnosis of white-coat hypertension.

A screening test should not only have a high specificity and negative predictive value, but high sensitivity as well. We investigated these characteristics of self-measured BP in 247 previously untreated hypertensive patients randomized in the THOP trial. ${ }^{23}$ Taking daytime ambulatory BP (time-weighted mean of readings obtained between 10 am and $8 \mathrm{pm}$ ) as the reference method to diagnose white-coat hypertension, we noticed that self-measured BP at home had a high specificity (89\%) and negative predictive value $(97 \%)$, but low sensitivity (68\%). Stergiou and colleagues 40,42 used a similar approach in a study of 133 untreated patients with high office BP and reported similar findings (specificity $83 \%$, negative predictive value $75 \%$, sensitivity $61 \%$ ). In both studies the agreement between home and ambulatory $\mathrm{BP}$ in the detection of white-coat hypertension was assessed using the kappa statistic. Reported kappa values for these studies were 0.38 and 0.41 , respectively, suggesting only low to moderate agreement. Taken together, the THOP study and that of Stergiou et al. suggest that the sensitivity of the self-measured home BP is low in the detection of truly hypertensive patients, but that home BP and ambulatory monitoring are complementary diagnostic methods.

\section{Home BP and the Management of Hypertension}

\section{Evidence from the THOP trial}

The THOP trial 2,43 aimed to determine whether or not long-term antihypertensive treatment based on self-measurement of BP at home might be more beneficial to the patient than treatment based on conventional BP measurement in a doctor's office.

After a 1-month run-in period, eligible hypertensive patients, those with a sitting diastolic office BP $>95 \mathrm{~mm} \mathrm{Hg}$, were randomized into two groups. In one group antihypertensive treatment was guided by the diastolic office BP as an average of three sitting readings obtained by a doctor in the office using a sphygmomanometer and, in the other group, by the diastolic home BP as an average of all 42 sitting readings (3 morning and 3 evening for 7 consecutive days prior to the patient's visit to the doctor). The patients used the oscillometric Omron HEM-705CP device (Omron Inc., Kyoto, Japan). Regardless of randomization, both home and office BP were measured in all patients at each visit. At baseline and after 6 and 12 months of randomized treatment, the ambulatory BP was also registered with an oscillometric Spacelabs 90207 recorder, but these measurements were not considered in any treatment decisions.

After randomization, the same standardized stepwise treatment regimen was applied in both groups to reach the same target diastolic BP ( $80 \mathrm{~mm} \mathrm{Hg}$ to $89 \mathrm{~mm} \mathrm{Hg}$ ). Follow-up visits were scheduled after $1,2,4,6,8,10$, and 12 months. One physician at the coordinating center blinded with regard to randomization made all treatment decisions based either on home or office BP. If the diastolic BP guiding treatment was above target $(>89 \mathrm{~mm} \mathrm{Hg})$, medical treatment was intensified by one step. If this diastolic BP was within the target range ( 80 to $89 \mathrm{~mm} \mathrm{Hg}$ ), medical treatment was left unchanged. If the diastolic BP guiding treatment was below the target range $(<80 \mathrm{~mm} \mathrm{Hg})$, medical treatment was reduced by one step and, for patients at the first step of treatment, this meant discontinuation of antihypertensive drug treatment. 


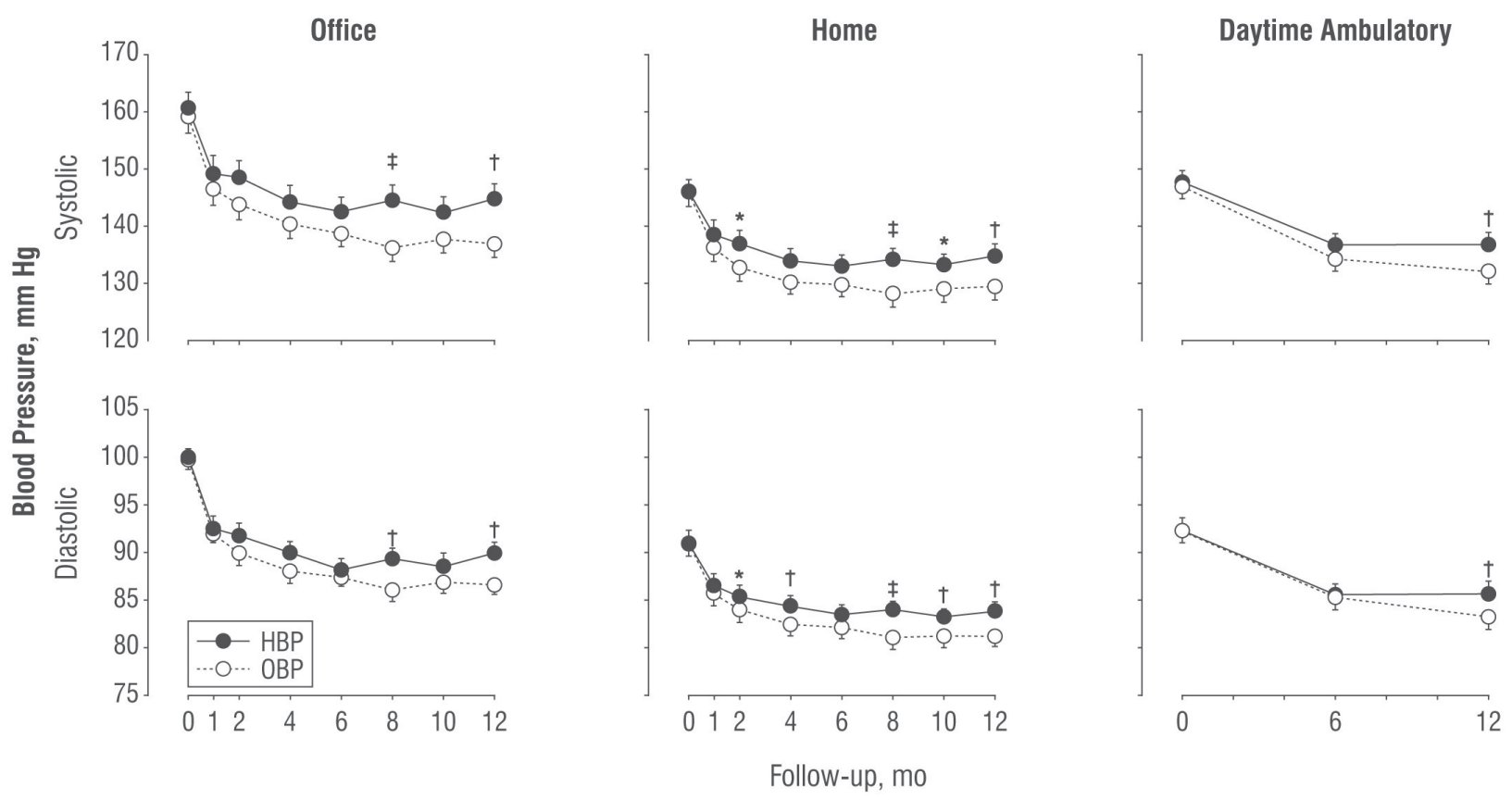

Figure 1. Mean systolic and diastolic office BP, home BP and daytime ambulatory BP. Error bars indicate standard errors. $P$-values are for the differences between patients randomized to treatment based on office BP (OBP) versus home BP (HBP). Asterisk indicates $P \leq 0.05$, dagger $P \leq 0.01$ and double dagger $P \leq 0.001$. (Reprinted with permission. Staessen et al.; Treatment of Hypertension Based on Home or Office Blood Pressure (THOP) Trial Investigators. Antihypertensive treatment based on blood pressure measurement at home or in the physician's office: a randomized controlled trial. JAMA 2004;291:955-964. Copyright 2004 American Medical Association. All rights reserved.)

Four hundred $(66 \%)$ of the 606 enrolled patients met the entry criteria and were randomized either to the office BP $(n=197)$ or home BP $(n=203)$ group. At baseline, both the office and home BP groups had similar characteristics and $\mathrm{BP}$ values. The median follow-up time was 350 days.

The office, home, and ambulatory BP values significantly decreased after randomization in both treatment groups (figure 1). After 1 month of randomized treatment, these decreases were similar in the office and home BP groups. Office BP measurements decreased an average of 12.6/8.2 $\mathrm{mm} \mathrm{Hg}$ in the office BP management group and 11.5/8.1 mm Hg in the home BP management group. Self-measured home BP decreased an average of $9.4 / 5.8 \mathrm{~mm} \mathrm{Hg}$ for the office BP management group and 7.5/4.8 $\mathrm{mm} \mathrm{Hg}$ for the home BP management group. After 6 months of treatment, these decreases in BP were still the same magnitude in the two randomized groups. Thereafter BP reductions became consistently and significantly greater in the office BP patients than in the home BP patients, leading to a final reduction in office $\mathrm{BP}$ of $22.0 / 14.0 \mathrm{~mm} \mathrm{Hg}$ in the office BP group and 15.3/10.5 mm Hg in the home BP group. The final differences between the two treatment groups thus were 6.8 and $3.5 \mathrm{~mm} \mathrm{Hg}$ for systolic and diastolic office BP, 4.9 and $2.9 \mathrm{~mm} \mathrm{Hg}$ for systolic and diastolic home BP, and 5.3 and $3.2 \mathrm{~mm} \mathrm{Hg}$ for systolic and diastolic daytime ambulatory BP (all differences between groups were $<0.001$ ).
More patients in the home BP group than in the office BP group $(25.6 \%$ and $11.3 \%$, respectively) were able to permanently stop antihypertensive treatment, but the opposite trend was observed for patients proceeding to multiple drug treatment $(38.7 \%$ in the home BP group and $45.1 \%$ in the office BP group).

Over the entire follow-up, the mean symptom score decreased on a 5-point scale from 1.52 to 1.40 in the office BP group and from 1.60 to 1.50 in the home BP group, leading to similar baseline-adjusted changes in symptom scores at the end of the trial in both groups. In both treatment groups there were similar significant decreases in electrocardiographic indexes of left ventricular mass: -0.03 $\mathrm{mV}$ for the R-wave in AvL in both the office BP and home BP groups; $-0.12 \mathrm{mV}$ and $-0.09 \mathrm{mV}$ for the Sokolow-Lyon index in the office BP and home BP groups, respectively; $-14 \mathrm{mV}$ and $-12 \mathrm{mV}$ for the Cornell voltage in the office BP and home $\mathrm{BP}$ groups, respectively; and $-13 \mu \mathrm{V}$ and $-12 \mu \mathrm{V}$ in the office BP and home BP groups, respectively, for the Cornell product.

From the above, we concluded that adjustment of antihypertensive treatment based on home BP instead of office BP led to less intensive drug treatment, but also to less BP control for the long-term with no differences in general well-being and electrocardiographic indexes of left ventricular mass. Furthermore, compared to repeated office 
White-coat hypertension

(lsolated clinic hypertension)
Masked hypertension

(Isolated am bulatory or

home hypertension)

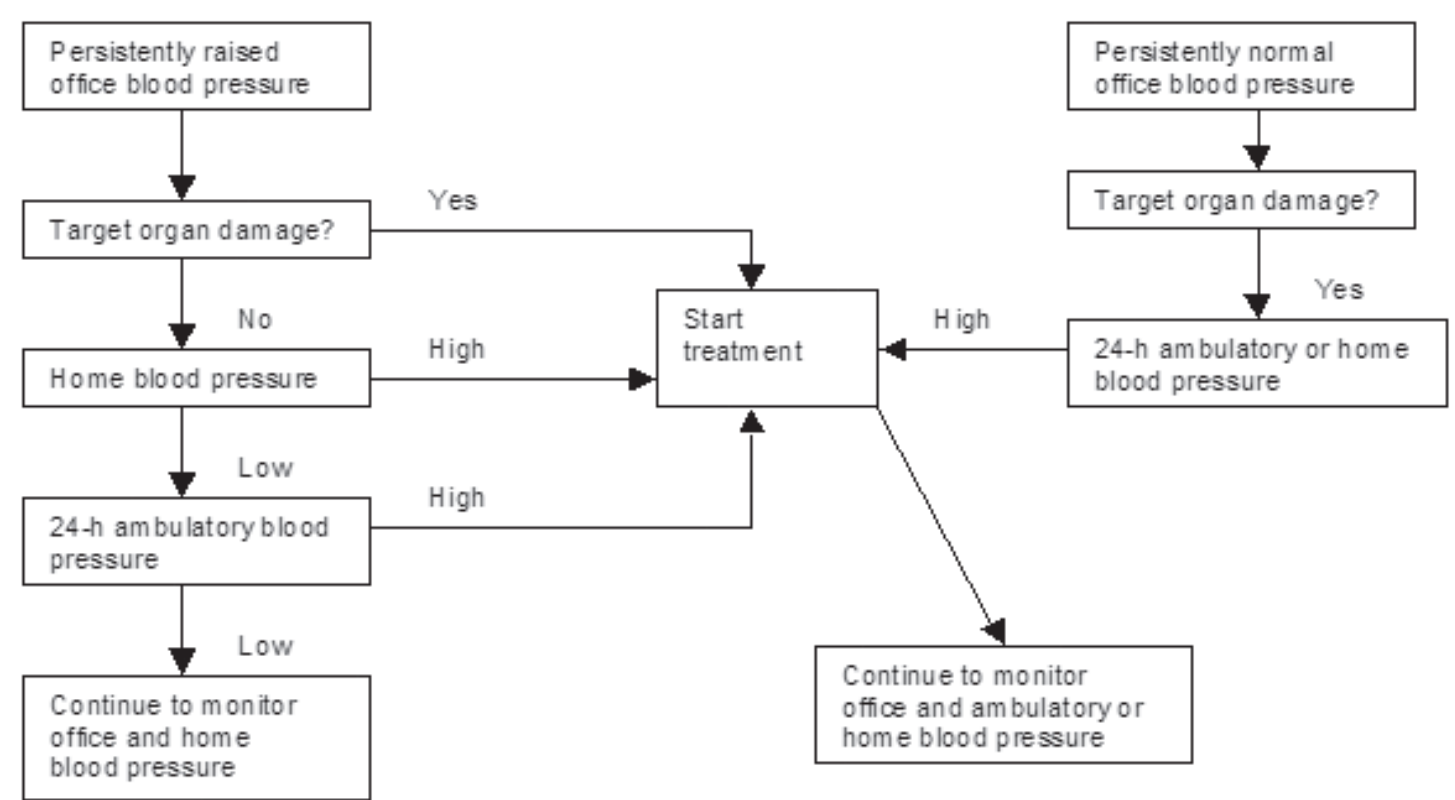

Figure 2. A possible strategy for the assessment of patients by use of office, home, and ambulatory BP.41 (Adapted with permission from Elsevier. Staessen et al. Essential hypertension. Lancet 2003;361:1629-1641.)

BP measurement, self-measurement of BP at home allowed the discontinuation of antihypertensive drugs in twice as many patients and thus helped to identify patients with white-coat hypertension.

\section{Recommendations for clinicians}

Although a recent Netherlands trial with a very similar design, the HOMERUS trial ${ }^{44}$ (Home versus Office Measurements, Reduction of Unnecessary treatment Study), has been initiated, to the best of our knowledge the THOP trial is still the only randomized, controlled trial that compared office and home BP for the initiation and titration of antihypertensive drug treatment.

The findings of the THOP trial do not support the hypothesis that self-measurement of BP, usually at the patient's home, might be a better guide to antihypertensive drug treatment than conventional BP measurement at the doctor's office. Office BP measurement should remain the standard for evaluating BP. In patients with raised office BP and either target organ damage or a high cardiovascular risk profile, treatment can be started without confirmation of the elevated office BP by automated BP measurement. In patients with high office BP but no target organ damage (suspected white-coat hypertension), or with normal office BP but unexplained target organ damage (suspected masked hypertension), ambulatory BP monitoring, home BP monitoring, or both must be used to confirm or refute the diagnosis (figure 2), whereas office BP measurements should be ignored. In the absence of other cardiovascular risk factors and signs of target organ damage, therapy of patients with white-coat hypertension may be limited to lifestyle measures and annual follow-up of the ambulatory BP. ${ }^{1}$

\section{Perspectives}

More long-term prospective outcome trials are needed to confirm the prognostic significance of home BP measurement and to firmly establish normal values, including the operational thresholds at which drug treatment should be instituted or can be discontinued. With this purpose, in May 2001 the HOMED-BP (Hypertension Objective treatment based on Measurement by Electrical Devices of Blood Pressure) study pilot trial was launched in Japan. ${ }^{45,46}$ The primary objective of this ongoing trial is to determine the optimal target BP level based on home BP. Patients will be randomized to one of two home BP target groups (home BP 134-125/84-80 mm Hg or $<125 / 80 \mathrm{~mm} \mathrm{Hg}$ ). The primary study outcome is the composite of non-fatal stroke, non-fatal myocardial infarction, and cardiovascular death. Patients will be recruited until February 2006 and followed for 7 years.

Furthermore and similar to our follow-up study of previous APTH (Ambulatory Blood Pressure Monitoring and Treatment of Hypertension) patients ${ }^{47}$ which showed that 
daytime ambulatory BP refined the prediction of major cardiovascular events over and beyond office BP, we also plan to obtain prognostic data in our THOP study population in the near future. The main objectives of the follow-up study will be to assess outcome in terms of office BP control and to compare the prognostic significance of baseline office, home, and ambulatory BP. Follow-up data to be collected will include information on vital status, cause of death, incidence of major cardiovascular and noncardiovascular diseases, treatment status and achieved office BP levels. For each patient, information will be obtained via a standard questionnaire to be completed by his or her treating physician.

Until more prospective data become available, conventional measurements at a doctor's office remain the standard for evaluating BP. Self-measurement at home and ambulatory monitoring increase the diagnostic accuracy of conventional BP measurement and allow identification of patients with white-coat or masked hypertension. Management of hypertension exclusively based on home BP measurement cannot be recommended.

\section{References}

1. Staessen JA, Wang J, Bianchi G, Birkenhager WH. Essential hypertension. Lancet 2003;361:1629-1641.

2. Staessen JA, Den Hond E, Celis H, Fagard R, Keary L, Vandenhoven G, O'Brien ET; Treatment of Hypertension Based on Home or Office Blood Pressure (THOP) Trial Investigators. Antihypertensive treatment based on blood pressure measurement at home or in the physician's office: a randomized controlled trial. JAMA 2004;291:955-964.

3. O’Brien E, Asmar R, Beilin L, Imai Y, Mallion JM, Mancia G, Mengden T, Myers M, Padfield P, Palatini P, Parati G, Pickering T, Redon J, Staessen J, Stergiou G, Verdecchia P; European Society of Hypertension Working Group on Blood Pressure Monitoring. European Society of Hypertension recommendations for conventional, ambulatory and home blood pressure measurement. J Hypertens 2003;21:821-848.

4. European Society of Hypertension-European Society of Cardiology Guidelines Committee. 2003 European Society of Hypertension-European Society of Cardiology guidelines for the management of arterial hypertension. J Hypertens 2003;21:1011-1053.

5. Imai Y, Otsuka K, Kawano Y, Shimada K, Hayashi H, Tochikubo O, Miyakawa M, Fukiyama K; Japanese Society of Hypertension. Japanese society of hypertension (JSH) guidelines for self-monitoring of blood pressure at home. Hypertens Res 2003;26:771-782.

6. Williams B, Poulter NR, Brown MJ, Davis M, McInnes GT, Potter JF, Sever PS, McG Thom S; British Hypertension Society. Guidelines for management of hypertension: report of the fourth working party of the British Hypertension Society, 2004-BHS IV. J Hum Hypertens 2004;18:139-185.

7. O'Brien E, Pickering T, Asmar R, Myers M, Parati G, Staessen J, Mengden T, Imai Y, Waeber B, Palatini P, Gerin W; Working Group on Blood Pressure Monitoring of the European Society of Hypertension. Working Group on Blood Pressure Monitoring of the European Society of Hypertension International Protocol for validation of blood pressure measuring devices in adults. Blood Press Monit 2002;7:3-17.
8. O’Brien E, Waeber B, Parati G, Staessen J, Myers MG. Blood pressure measuring devices: recommendations of the European Society of Hypertension. BMJ 2001;322:531-536.

9. Parati G, Bilo G, Mancia G. Blood pressure measurement in research and in clinical practice: recent evidence. Curr Opin Nephrol Hypertens 2004;13:343-357.

10. Parati G, Asmar R, Stergiou GS. Self blood pressure monitoring at home by wrist devices: a reliable approach? J Hypertens 2002;20:573-578.

11. Kikuya M, Chonan K, Imai Y, Goto E, Ishii M; Research Group to Assess the Validity of Automated Blood Pressure Measurement Devices in Japan. Accuracy and reliability of wrist-cuff devices for self-measurement of blood pressure. J Hypertens 2002;20:629-638.

12. Shahriari M, Rotenberg DK, Nielsen JK, Wiinberg N, Nielsen PE. Measurement of arm blood pressure using different oscillometry manometers compared to auscultatory readings. Blood Press 2003;12:155-159.

13. Caridi G, Zoccali C, Enia G. The oscillometric wrist devices: an estimate of the 'supine' error. J Hum Hypertens 2002; 16:525-526.

14. Uen S, Weisser B, Wieneke P, Vetter H, Mengden T. Evaluation of the performance of a wrist blood pressure measuring device with a position sensor compared to ambulatory 24-hour blood pressure measurements. Am J Hypertens 2002;15:787-792.

15. Braam RL, Asian B, Thien T. Oscillometric wrist blood pressure measuring devices. Neth J Med 2003;61:313-317.

16. Parati G, Stergiou G. Self blood pressure measurement at home: how many times? J Hypertens 2004;22:1075-1079.

17. Chatellier G, Day M, Bobrie G, Menard J. Feasibility study of $\mathrm{N}$-of-1 trials with blood pressure self-monitoring in hypertension. Hypertension 1995;25:294-301.

18. Imai Y, Ohkubo T, Hozawa A, Tsuji I, Matsubara M, Araki T, Chonan K, Kikuya M, Satoh H, Hisamichi S, Nagai K. Usefulness of home blood pressure measurements in assessing the effect of treatment in a single-blind placebo-controlled open trial. J Hypertens 2001;19:179-185.

19. Chatellier G, Dutrey-Dupagne C, Vaur L, Zannad F, Genes N, Elkik F, Menard J. Home self blood pressure measurement in general practice. The SMART study. Self-measurement for the Assessment of the Response to Trandolapril. Am J Hypertens 1996;9:644-652.

20. Stergiou GS, Skeva II, Zourbaki AS, Mountokalakis TD. Self-monitoring of blood pressure at home: how many measurements are needed? J Hypertens 1998;16:725-731.

21. Stergiou GS, Baibas NM, Gantzarou AP, Skeva II, Kalkana CB, Roussias LG, Mountokalakis TD. Reproducibility of home, ambulatory, and clinic blood pressure: implications for the design of trials for the assessment of antihypertensive drug efficacy. Am J Hypertens 2002;15:101-104.

22. Celis H, De Cort P, Fagard R, Thijs L, Staessen JA. For how many days should blood pressure be measured at home in older patients before steady levels are obtained? J Hum Hypertens 1997;11:673-677.

23. Hond ED, Celis H, Fagard R, Keary L, Leeman M, O’Brien E, Vandenhoven G, Staessen JA; THOP investigators. Self-measured versus ambulatory blood pressure in the diagnosis of hypertension. J Hypertens 2003;21:717-722.

24. Brook RD. Home blood pressure: accuracy is independent of monitoring schedules. Am J Hypertens 2000;13:625-631.

25. Ohkubo T, Asayama K, Kikuya M, Metoki H, Hoshi H, Hashimoto J, Totsune K, Satoh H, Imai Y; Ohasama Study. How many times should blood pressure be measured at home for better prediction of stroke risk? Ten-year follow-up results from the Ohasama study. J Hypertens 2004;22:1099-1104. 
26. Mejia AD, Julius S, Jones KA, Schork NJ, Kneisley J. The Tecumseh Blood Pressure Study. Normative data on blood pressure self-determination. Arch Intern Med 1990;150:1209-1213.

27. de Gaudemaris R, Chau NP, Mallion JM. Home blood pressure: variability, comparison with office readings and proposal for reference values. Groupe de la Mesure, French Society of Hypertension. J Hypertens 1994;12:831-838.

28. Weisser B, Grune S, Burger R, Blickenstorfer H, Iseli J, Michelsen SH, Opravil R, Rageth S, Sturzenegger ER, Walker P, Widmer W, Vetter W. The Dubendorf Study: a population-based investigation on normal values of blood pressure self-measurement. J Hum Hypertens 1994;8:227-231.

29. Mancia G, Sega R, Bravi C, De Vito G, Valagussa F, Cesana G, Zanchetti A. Ambulatory blood pressure normality: results from the PAMELA study. J Hypertens 1995;13):1377-1390.

30. Thijs L, Staessen JA, Celis H, de Gaudemaris R, Imai Y, Julius S, Fagard R. Reference values for self-recorded blood pressure: a meta-analysis of summary data. Arch Intern Med 1998; 158:481-488.

31. Thijs L, Staessen JA, Celis H, Fagard R, De Cort P, de Gaudemaris R, Enstrom I, Imai Y, Julius S, Menard J, Mion D, Palatini P, Rosenfeld J, Shapiro D, Spence D, Stergiou G. The international database of self-recorded blood pressures in normotensive and untreated hypertensive subjects. Blood Press Monit 1999;4:77-86.

32. Stergiou GS, Thomopoulou GC, Skeva II, Mountokalakis TD. Home blood pressure normalcy: the Didima study. Am J Hypertens 2000;13:678-685.

33. Ohkubo T, Imai Y, Tsuji I, Nagai K, Kato J, Kikuchi N, Nishiyama A, Aihara A, Sekino M, Kikuya M, Ito S, Satoh $\mathrm{H}$, Hisamichi S. Home blood pressure measurement has a stronger predictive power for mortality than does screening blood pressure measurement: a population-based observation in Ohasama, Japan. J Hypertens 1998;16:971-975.

34. Tsuji I, Imai Y, Nagai K, Ohkubo T, Watanabe N, Minami N, Itoh O, Bando T, Sakuma M, Fukao A, Satoh H, Hisamichi $\mathrm{S}$, Abe K. Proposal of reference values for home blood pressure measurement: prognostic criteria based on a prospective observation of the general population in Ohasama, Japan. Am J Hypertens 1997;10:409-418.

35. Bobrie G, Chatellier G, Genes N, Clerson P, Vaur L, Vaisse B, Menard J, Mallion JM. Cardiovascular prognosis of "masked hypertension" detected by blood pressure self-measurement in elderly treated hypertensive patients. JAMA 2004;291:1342-1349.

36. Bobrie G, Genes N, Vaur L, Clerson P, Vaisse B, Mallion JM, Chatellier G. Is "isolated home" hypertension as opposed to "isolated office" hypertension a sign of greater cardiovascular risk? Arch Intern Med 2001;161:2205-2211.

37. Mallion JM, Genes N, Vaur L, Clerson P, Vaisse B, Bobrie G, Chatellier G. Blood pressure levels, risk factors and antihypertensive treatments: lessons from the SHEAF study. J Hum Hypertens 2001;15:841-848.

38. Yarows SA, Staessen JA. How to use home blood pressure monitors in clinical practice. Am J Hypertens 2002;15:93-96.

39. Chobanian AV, Bakris GL, Black HR, Cushman WC, Green LA, Izzo JL Jr, Jones DW, Materson BJ, Oparil S, Wright JT Jr, Roccella EJ; National Heart, Lung, and Blood Institute Joint National Committee on Prevention, Detection, Evaluation, and Treatment of High Blood Pressure; National High Blood Pressure Education Program Coordinating Committee. The Seventh Report of the Joint National Committee on Prevention, Detection, Evaluation, and Treatment of High Blood Pressure: the JNC 7 report. JAMA 2003;289:2560-2572.
40. Stergiou GS, Alamara CV, Skeva II, Mountokalakis TD. Diagnostic value of strategy for the detection of white coat hypertension based on ambulatory and home blood pressure monitoring. J Hum Hypertens 2004;18:85-89.

41. Pickering TG. Self-monitoring of blood pressure. In: Ambulatory monitoring and blood pressure variability (part 1). London: Science Press; 1990. 8.5.

42. Stergiou GS, Skeva II, Baibas NM, Kalkana CB, Roussias LG, Mountokalakis TD. Diagnosis of hypertension using home or ambulatory blood pressure monitoring: comparison with the conventional strategy based on repeated clinic blood pressure measurements. J Hypertens 2000;18:1745-1751.

43. Celis H, Staessen JA, Buntinx F, Fagard RH, Leeman M, Thijs L, Van Hedent T. Antihypertensive treatment based on home or office blood pressure measurement: protocol of the randomized controlled THOP trial. Blood Press Monit 1998;3:S29-S35.

44. Verberk WJ, Kroon AA, Kessels AG, Dirksen C, Nelemans PJ, Lenders JW, Thien TA, van Montfrans GA, Smit AJ, de Leeuw PW. Home versus Office blood pressure MEasurements: Reduction of Unnecessary treatment Study: rationale and study design of the HOMERUS trial. Blood Press 2003;12:326-333.

45. Fujiwara T, Matsubara M, Ohkubo T, Imai Y. Study design of HOMED-BP: hypertension objective treatment based on measurement by electrical devices of blood pressure. Clin Exp Hypertens 2003;25:143-144.

46. Aoki Y, Asayama K, Ohkubo T, Nishimura T, Kikuya M, Metoki H, Hozawa A, Hashimoto J, Michimata M, Matsubara M, Araki T, Imai Y; HOMED-BP Study Group. Progress report on the HOMED-BP Study: hypertension objective treatment based on measurement by electrical devices of blood pressure study. Clin Exp Hypertens 2004;26:119-127.

47. Celis H, Staessen JA, Thijs L, Buntinx F, De Buyzere M, Den Hond E, Fagard RH, O’Brien ET; Ambulatory Blood Pressure and Treatment of Hypertension Trial Investigators. Cardiovascular risk in white-coat and sustained hypertensive patients. Blood Press 2002;11:352-356.

\section{Author Affiliations}

Hilde Celis, MD, Elly Den Hond, PhD, Jan A. Staessen, MD, PhD, FAHA, Study Coordinating Center, Hypertension and Cardiovascular Rehabilitation Unit, Department of Molecular and Cardiovascular Research, University of Leuven, Leuven, Belgium 